\title{
Pituitary magnetic resonance imaging predictive role in the therapeutic response of growth hormone-secreting pituitary adenomas
}

\author{
Fabio Tortora ${ }^{1}$, Alberto Negro ${ }^{1}$, Ludovica F. S. Grasso ${ }^{2}$, Annamaria Colao ${ }^{2}$, Rosario Pivonello ${ }^{2}$, \\ Alessandra Splendiani ${ }^{3}$, Luca Brunese ${ }^{4}$, Ferdinando Caranci ${ }^{4}$
}

${ }^{1}$ Department of Precision Medicine, School of Medicine, University of Campania “Luigi Vanvitelli”, Naples, Italy; ${ }^{2}$ Dipartimento di Medicina Clinica e Chirurgia, Sezione di Endocrinologia, Università Federico II di Napoli, Naples, Italy; ${ }^{3}$ Department of Biotechnology and Applied Clinical Sciences, University of L'Aquila, L’Aquila, Italy; ${ }^{4}$ Department of Medicine and Health Sciences "V. Tiberio", University of Molise, Campobasso, Italy Contributions: (I) Conception and design: F Tortora, F Caranci; (II) Administrative support: F Tortora, F Caranci; (III) Provision of study materials: All authors; (IV) Collection and assembly of data: All authors; (V) Data analysis and interpretation: All authors; (VI) Manuscript writing: All authors; (VII) Final approval of manuscript: All authors.

Correspondence to: Prof. Ferdinando Caranci. Department of Medicine and Health Sciences "V. Tiberio", University of Molise, Via Francesco De Sanctis 1, 86100 Campobasso, Italy. Email: ferdinando.caranci@unimol.it.

Background: Growth hormone (GH)-secreting pituitary adenomas, responsible for the development of acromegaly, are the second most frequent type of secreting pituitary adenomas and are characterized by very variable T2-weighted signal intensity on pituitary magnetic resonance imaging (MRI). Previous data have demonstrated a correlation between T2-weighted tumor signal intensity and response to therapy with conventional somatostatin analogs (SSA) in patients with acromegaly. The aim of the current retrospective study was to investigate the correlation between the T2-weighted tumor signal on pituitary MRI and both biochemical and radiological response to first-line SSA therapy.

Methods: Twenty-two naive patients with acromegaly were eligible for the study (14 females and 8 males, mean age \pm SD: $58.8 \pm 15.74$ ). A biochemical evaluation (GH and IGF-I levels) and an MRI assessment (volume and signal intensity analysis of adenoma) were conducted in each patient at diagnosis and after 12 months of SSA therapy.

Results: On diagnostic pituitary MRI, 16 (72.7\%) adenomas were T2- hypointense and 6 (27.2\%) T2hyperintense. After 12 months of SSA therapy, IGF-I levels decreased by more than $50 \%$ from baseline in $62.5 \%$ of patients with T2-hypointense and $33.3 \%$ of patients with T2- hyperintense tumor signal, respectively $(\mathrm{P}=0.03)$. Moreover, $\mathrm{GH}$ levels decreased by more than $80 \%$ from baseline in $81.3 \%$ and $33.3 \%$ of patients with $\mathrm{T} 2$-hypointense and $\mathrm{T} 2$-hyperintense tumor signal $(\mathrm{P}=0.02)$. A significant tumor volume reduction ( $\geq 20 \%$ ) was observed in $75 \%$ of the $\mathrm{T} 2$-hypointense and $33.3 \%$ of the T2-hyperintense adenomas $(\mathrm{P}=0.001)$.

Conclusions: In naive patients with acromegaly, first-line SSA therapy is associated with a better biochemical response and greater tumor shrinkage in T2-hypointense compared to T2-hyperintense adenomas. Therefore, T2-weighted sequences of pituitary MRI can help to classify GH-secreting pituitary adenomas into a T2-hypointense and T2-hyperintense type and, therefore, to identify patients who can better respond to first-line SSA therapy.

Keywords: Acromegaly; pituitary adenoma; magnetic resonance imaging (MRI); T2-weighted signal; somatostatin analogues (SSA)

Submitted Jan 20, 2019. Accepted for publication Jun 05, 2019.

doi: 10.21037 /gs.2019.06.04

View this article at: http://dx.doi.org/10.21037/gs.2019.06.04 


\section{Introduction}

Acromegaly is characterized by increased release of growth hormone $(\mathrm{GH})$ and, consequently, insulin-like growth factor I (IGF-I), generally because of a GH-secreting pituitary adenoma (1). GH-secreting adenomas are the second most frequent type of secreting pituitary adenomas $(2,3)$ and usually present as macroadenomas (maximal diameter equal or greater than $10 \mathrm{~mm}$ ) on magnetic resonance imaging (MRI) (4). Compared with different types of pituitary adenomas (i.e., prolactinomas), which are usually hyperintense on the $\mathrm{T} 2$-weighted sequences of pituitary MRI $(5,6)$, the T2-weighted signal intensity of GH-secreting adenomas is variable. The T2-weighted signal intensity of $\mathrm{GH}$-secreting pituitary adenomas seems to be an important predictor of response of medical therapy with conventional somatostatin analogs (SSA) in acromegalic patients; however, the available data are still unclear, since many studies suffered from low-quality images or the absence of T2-weighted sequences at the pituitary MRI. The main predictors of responsiveness to SSA therapy include gender, age, baseline GH and IGF-I levels, baseline tumor volume and somatostatin receptor (SSTR) expression, particularly the SSTR2 and SSTR5 subtypes (7-9). Moreover, on the basis of pathological analysis, the granulation pattern seems to predict the response to SSA therapy, with densely granulated better responding to SSA therapy, compared with the more common sparsely granulated adenomas (10). Furthermore, immunohistochemistry and immunohistochemical markers can better define aggressive pituitary adenomas, which hardly respond to SSA therapy $(11,12)$. However, despite pathological analysis contributes in giving important information on aggressiveness of pituitary adenomas and response to SSA therapy, it has the limitation to be available only after surgery. Therefore, at the time of diagnosis this information cannot be used to predict the response to firstline SSA therapy.

Pituitary MRI resolution has improved in recent years and represents a central part of the diagnostic work-up of patients with acromegaly. As a noninvasive diagnostic technique, high-resolution pituitary MRI could be useful in helping to characterize GH-secreting pituitary adenomas and predict subsequent clinical behavior and pathological features. Recent studies demonstrated in GH-secreting pituitary adenomas that the presence at the pituitary MRI of T2-hypointense signal, which correlates with dense tumor granularity, seems to predict a favorable response to SSA therapy $(5,13,14)$. In particular, lower T2-weighted signal intensity correlated with a better response to SSA therapy, used before (14) or after surgery (10), in terms of reduction of GH and IGF-I levels, as well as with a greater tumor shrinkage.

The identification of new predictive markers of response to medical therapy is mandatory to improve the management of patients with acromegaly.

The current study describes a retrospective analysis in a population of 22 acromegalic patients, with the aim to clarify the relationship between the T2-weighted tumor signal at the pituitary MRI and both the hormonal and radiological response to 12 months first-line SSA therapy.

\section{Methods}

\section{Patients population}

Twenty-two patients with acromegaly (14 females and 8 males, mean age \pm SD: $58.8 \pm 15.74$ ), admitted at Neuroendocrine Disease Unit of the Federico II University and whose diagnostic pituitary MRI was evaluated in the Neuroradiology Unit of "Luigi Vanvitelli" University of Naples between July 2016 and February 2017, were included in the current study.

The biochemical diagnosis of acromegaly was based on the following criteria: (I) lack of GH suppression below $1.0 \mu \mathrm{g} / \mathrm{L}$ after an oral glucose tolerance test (OGTT); (II) IGF-I levels above the normal range adjusted for age.

The inclusion criteria were the following:

(I) Presence of GH-secreting pituitary adenoma on diagnostic MRI (maximal diameter greater than $5 \mathrm{~mm})$;

(II) Previous treatment with SSA for at least 12 months used as first-line therapy;

(III) Availability of hormonal profile and pituitary MRI at the diagnosis and 12 months after the starting of primary SSA treatment.

The exclusion criteria were the following:

(I) Previous pituitary surgery;

(II) Previous pituitary radiotherapy;

(III) Previous medical treatment with cabergoline and/or pegvisomant.

\section{Biochemical evaluation}

GH and IGF-I levels were evaluated in each patient at diagnosis and after 12 months of SSA therapy. The 

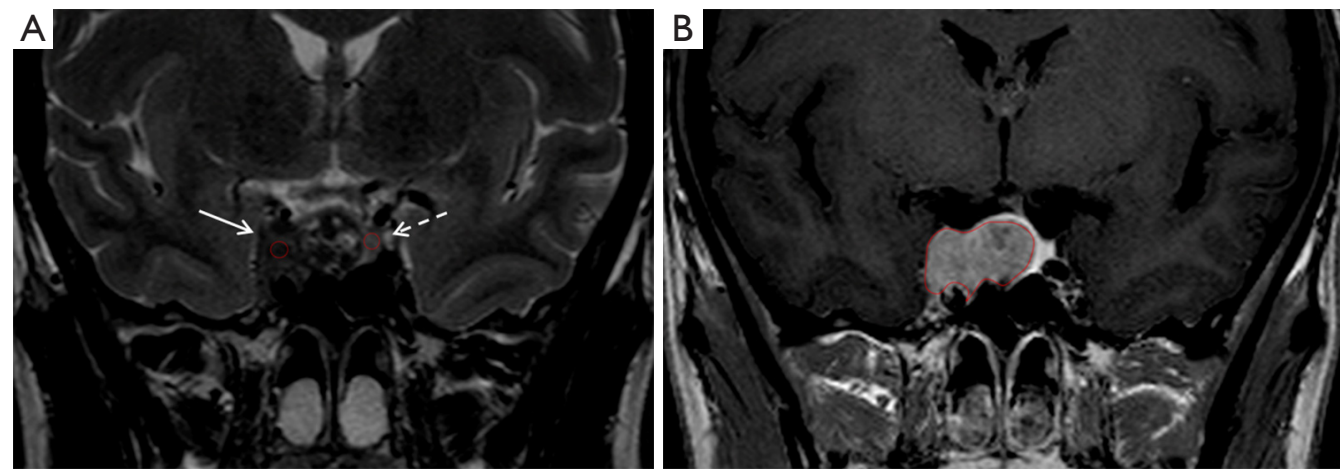

Figure 1 Adenoma characteristics based on the signal intensity. (A) T2-weighted coronal image. GH-secreting macroadenoma infiltrating the right cavernous sinus: comparison between the average intensity level of the solid portion of the adenoma (white arrow) with the average intensity level of the healthy pituitary gland (dotted white arrow); (B) T1-weighted coronal image after contrast medium intravenous injection (late dynamic acquisition): manual segmentation of the whole adenoma. Comparison of the T2-weighted coronal image with the late dynamic coronal image, in order to ensure that the analyzed adenomatous portion is predominantly solid and to avoid the sampling of the colliquative or cystic portions. GH, growth hormone.

determination of serum hormone levels was obtained with the solid phase chemiluminescent immunometric method (IMMULITE 2000 hGH, Siemens, Erlangen, Germany) using calibrators recommended by the WHO (IS 98/574 for hGH and IS $87 / 518$ for IGF-1). The specific IGF1 normal range of patient's reference laboratory was used to evaluate IGF-1 values and the response of each patient throughout the study.

\section{MRI assessment}

All patients' MRI studies were examined at the initial diagnosis and after 12 months with a 1.5 Tesla (T) scanner (Symphony, Siemens, Erlangen, Germany).

The following sequences were acquired:

(I) Turbo Spin-Eco T1 (TSE-T1) on the sagittal plane (TR: $500 \mathrm{~ms}$; TE: $15 \mathrm{~ms}$; Thickness: 3 mm; NEX: 2; reconstruction diameter $180 \mathrm{~mm}$; flip angle: $90^{\circ}$ );

(II) TSE-T1 on the coronal plane (TR: $500 \mathrm{~ms}$; TE: $15 \mathrm{~ms}$; Thickness: $3 \mathrm{~mm}$; NEX: 2; reconstruction diameter $180 \mathrm{~mm}$; flip angle: $90^{\circ}$ );

(III) TSE-T2 on the coronal plane (TR: 3,000 ms; TE: $100 \mathrm{~ms}$; Thickness: $3 \mathrm{~mm}$; NEX: 4; reconstruction diameter: $180 \mathrm{~mm}$; flip angle: $90^{\circ}$ );

(IV) Dynamic study during administration of paramagnetic contrast agent (gadolinium) in TSE-T1 sequence on the coronal plane repeated 5 times with $30 \mathrm{~s}$ intervals.

All the images were subsequently analyzed through the image communication and archiving system (OsiriX). Tumor volume was calculated through the use of specific OsiriX tools ("compute volume") after outlining the peripheral margins of the lesion with regions of interest (ROI) on each image, preferring those acquired with thinner thickness and on a coronal plane to obtain a more accurate analysis (12). The distinction between microadenoma (maximal diameter below $10 \mathrm{~mm}$ ) and macroadenoma (maximal diameter equal or above $10 \mathrm{~mm}$ ) was obtained by measuring the maximum transverse diameter of the adenoma on the coronal plane. In order to classify the adenomas based on the signal intensity, the average intensity level of the solid portion of the adenoma was compared with the average intensity level of the healthy pituitary gland in the T2-weighted sequences on the coronal plane (Figure 1A) (15), or, when this was not possible, with a reference tissue (16), represented by temporal grey matter, outlining a circular ROI in a standardized area of the temporal grey matter, in accordance with previous studies demonstrating a strong correlation of the intensity of the adenoma signal with either white or grey temporal matter as a reference tissue (17). According with this methodology, the adenomas were divided into two groups basing on the signal intensity in the T2-weighted images at pituitary MRI (hypointense and hyperintense). In order to ensure that the analyzed adenomatous portion was solid, avoiding the sampling of the colliquative or cystic portions, the T2-weighted coronal image was compared with the late dynamic coronal images (Figure 1B), because of the prolonged permanence of the increased signal intensity, due to the distribution of the paramagnetic contrast agent 

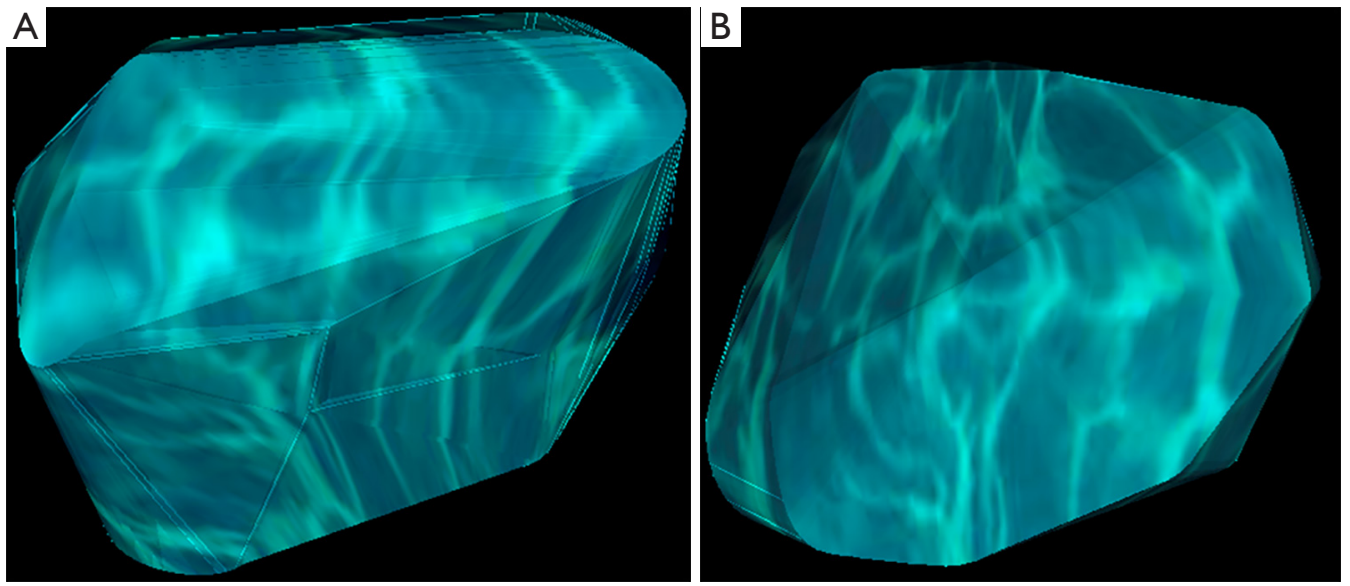

Figure 2 Example of the volumetric analysis. (A) Calculated volume $=5.0721 \mathrm{~cm}^{3}$ (mean 300.1103, SD 49.1470); (B) calculated volume (1-year control) $=3.0427 \mathrm{~cm}^{3}($ mean 286.4370, SD 43.0290).

in the adenoma cellular cordons, compared to the rapid normalization of healthy parenchymal portions (18). Finally, the degree of local regional infiltration and invasiveness of the adenoma was evaluated using the SIPAP score (19), according to the Knosp-Steiner classification (20) of parasellar growth, with the addition of a grading for superior, inferior, anterior, and posterior tumor extension (19).

\section{Evaluation of the response to the SSA treatment}

The biochemical response to SSA therapy was evaluated after 12 months from the beginning of therapy, by measuring GH and IGF-I and calculating the percent degree compared to the baseline examination. The radiological response to SSA therapy was evaluated after 12 months from the beginning of therapy, by measuring at MRI examination tumor volume and calculating the percent decrease compared to the baseline examination (for an example see Figure 2).

The following biochemical and radiological cut-offs, representative of a significant biochemical or radiological response, were used to evaluate the effect of SSA treatment (17):

(I) IGF-I value reduction $\geq 50 \%$;

(II) $\mathrm{GH}$ value reduction $\geq 80 \%$;

(III) Tumor volume reduction $\geq 20 \%$.

\section{Statistical analysis}

Data were analyzed using SPSS Software for Windows, version 22.0 (SPSS, Inc., Cary, NC, USA). Data are reported as mean $\pm \mathrm{SD}$, unless otherwise specified. The comparison between prevalence of different conditions before and after treatment was performed by $\chi^{2}$ test corrected by Fisher exact test when necessary. The correlation study was done by calculating the Pearson's correlation coefficients. Kruskall-Wallis test was used to evaluate the differences in clinical and biochemical parameters between two groups (patients with hypointense adenomas and those with hyperintense ones). Significance was set at $5 \%$.

\section{Results}

\section{Baseline characteristics}

At diagnosis, the median random GH level was $11.9 \mu \mathrm{g} / \mathrm{L}$ and the median IGF-I level was $686 \mathrm{ng} / \mathrm{mL}$. The baseline characteristics and biochemical data of the 22 patients are reported in Table 1. According to the tumor signal intensity on diagnostic MRI, the 22 patients resulted in $16(72.7 \%)$ patients with hypointense and $6(27.2 \%)$ patients with hyperintense adenomas (for an example see Figure 3). No difference was found in the distribution of the clinical biochemical parameters between the two groups of patients.

\section{Evaluation of the response to the SSA treatment}

The biochemical and radiological response of SSA treatment was evaluated after 12 months of treatment (Tables 2,3). A significant response of IGF-I (IGF-I levels decreased $\geq 50 \%)$ was observed in $10(62.5 \%)$ patients with hypointense and $2(33.3 \%)$ patients with hyperintense 
adenomas $(\mathrm{P}=0.03)$. A significant response of $\mathrm{GH}(\mathrm{GH}$ levels decreased $\geq 80 \%$ ) was observed in $13(81.3 \%)$ patients with hypointense and $2(33.3 \%)$ patients with hyperintense adenomas $(\mathrm{P}=0.02)$. Patients with hypointense adenomas had mean GH and IGF-I levels decrease of $51 \%$ and $80.8 \%$, respectively, after 12 months of SSA treatment, compared with $11 \%$ and $29.5 \%$ of patients with hyperintense adenomas. A significant tumor volume reduction (tumor reduction $\geq 20 \%$ ) was observed in $12(75 \%)$ patients with hypointense and $2(33.3 \%)$ patients with hyperintense

Table 1 Patients characteristics and biochemical data at diagnosis $($ mean $\pm \mathrm{SD})$

\begin{tabular}{lcc}
\hline Groups characteristics & Hypointense & Hyperintense \\
\hline N (total population $=22)$ & 16 & 6 \\
Gender $(\mathrm{M} / \mathrm{F})$ & $6 / 10$ & $2 / 4$ \\
Age (years) & $58.8 \pm 15.17$ & $61.5 \pm 8.7$ \\
Serum GH $(\mu \mathrm{g} / \mathrm{L})$ & $12.17 \pm 9.93$ & $7.37 \pm 3.28$ \\
Serum IGF-1 $(\mu \mathrm{g} / \mathrm{L})$ & $653.43 \pm 290.96$ & $593.97 \pm 257.55$ \\
Tumor volume $\left(\mathrm{cm}^{3}\right)$ & $2.12 \pm 2.86$ & $3.12 \pm 2.84$ \\
\hline
\end{tabular}

*, Kruskal-Wallis test $(\mathrm{P}>0.05$ means not statistically significant differences of distribution). GH, growth hormone; IGF-1, insulinlike growth factor $I$. adenomas $(\mathrm{P}=0.001)$.

\section{Discussion}

Transsphenoidal surgery represents the first-line treatment for the majority of patients with acromegaly, except for patents with high surgical risk, patients who refuse surgery or patients who have invasive, unresectable tumors $(21,22)$. Treatment with conventional SSA is recommended as firstline therapy in patients who are not suitable for surgery or who are unlikely to be cured by surgery (21,23-29). The choice of treatment of acromegaly mainly depends on the size and invasiveness of the adenoma, patient's age and comorbidities. SSA therapy can also be used as a preoperative treatment in macroadenomas, with the aim of improving surgical outcome by inducing tumor shrinkage beyond preventing postoperative complications. The biochemical control of SSA therapy is reported in about $50-60 \%$ of patients, although large differences in the efficacy of SSA in controlling GH and IGF-I excess between different case series have been described (30-32). Moreover, SSA induce tumor shrinkage in up to $80 \%$ of patients, with reduction in tumor volume which are greater after first-line than post-surgical therapy $(33,34)$. However, approximately $25 \%$ of patients treated with SSA for at least
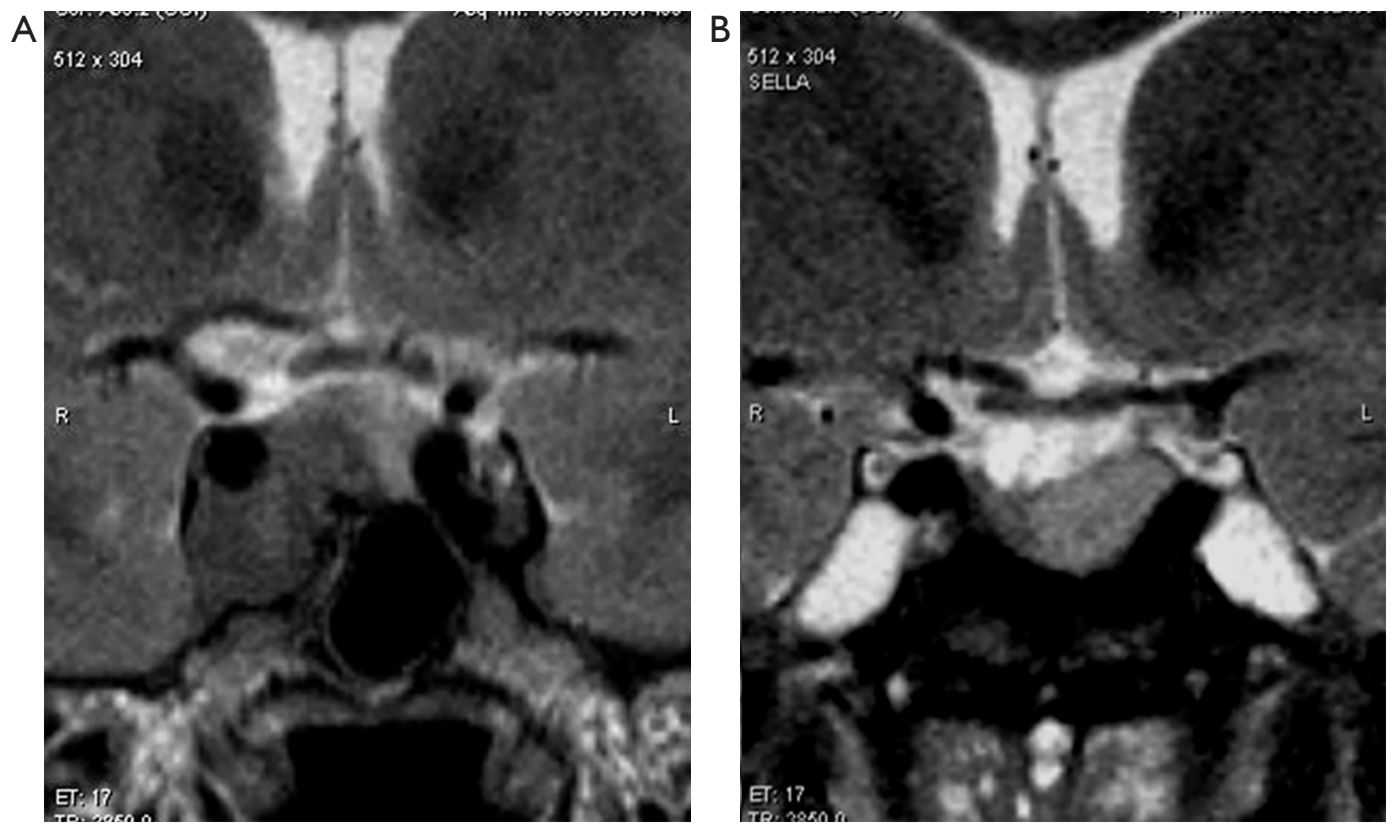

Figure 3 T2-weighted coronal image. (A) Hypointense growth hormon secreting adenoma; (B) hyperintense growth hormon secreting adenoma. 
Table 2 Biochemical and radiological response to SSA therapy: values at 12 months follow-up $($ mean $\pm \mathrm{SD})$

\begin{tabular}{lcc}
\hline Groups characteristics & Hypointense & Hyperintense \\
\hline N (total population $=22)$ & 16 & 6 \\
Gender $(\mathrm{M} / \mathrm{F})$ & $6 / 10$ & $2 / 4$ \\
Age $($ years $)$ & $58.8 \pm 15.17$ & $61.5 \pm 8.7$ \\
Serum GH $(\mu \mathrm{g} / \mathrm{L})$ & $2.33 \pm 3.14$ & $5.19 \pm 3.40$ \\
Serum IGF-1 $(\mu \mathrm{g} / \mathrm{L})$ & $316.50 \pm 256.09$ & $526.67 \pm 256.57$ \\
Tumor volume $\left(\mathrm{cm}^{3}\right)$ & $1.31 \pm 1.76$ & $2.80 \pm 2.87$ \\
\hline
\end{tabular}

*, Kruskal-Wallis test $(\mathrm{P}>0.05$ means not statistically significant differences of distribution). SSA, somatostatin analogs; $\mathrm{GH}$, growth hormone; IGF-1, insulin-like growth factor I.

Table 3 Biochemical and radiological response to SSA therapy: percentage of patients with significant response

\begin{tabular}{lcc}
\hline Groups characteristics & Hypointense (\%) & Hyperintense (\%) \\
\hline IGF-1 reduction & 51.60 & 11.30 \\
GH reduction & 80.85 & 29.50 \\
Volume reduction & 37.90 & 10.20 \\
\hline
\end{tabular}

*, P>0.05 means not statistically significant differences of distribution. SSA, somatostatin analogs; IGF-1, insulin-like growth factor I; GH, growth hormone.

12 months result to be resistant to SSA (35). The main predictors of response to SSA therapy include gender, age, baseline GH and IGF-I levels, tumor volume and SSTR expression. Recently, the T2-weighted signal intensity of the pituitary tumor on MRI images has been indicated as a potential predictor of response to SSA $(13,14)$. In particular, T2-hypointense adenomas correlated with a better response to SSA treatment in terms of reduction in GH and IGF-I levels and a greater tumor shrinkage (17).

The results of the current study confirm the usefulness of T2-weighted signal intensity of pituitary tumor on MRI images to identify patients who can better respond to firstline SSA therapy. In the current study, 16 (72.7\%) adenomas resulted hypointense whereas $6(27.2 \%)$ adenomas resulted hyperintense on diagnostic pituitary MRI. According with previous studies (13-15) patients with hypointense adenomas reported a significantly better biochemical and radiological response to first-line SSA therapy.

The association between T2-weighted signal intensity of the pituitary tumor and the response to SSA therapy in patients with acromegaly was firstly studied by Puig-
Domingo et al., reporting a significantly better hormonal response to SSA in hypointense than hyperintense adenomas after 6 months of therapy. In particular, IGF-I normalization was reported in $71 \%$ of patients with hypointense compared with $20 \%$ of patients with hyperintense adenomas. Moreover, IGF-I decreased by more than $50 \%$ from baseline values in $66 \%$ of patients with hypointense compared with $31 \%$ of patients with hyperintense adenomas (13).

Several additional groups studied the effect of first-line SSA therapy and its relationship to T2-weighted signal intensity of the pituitary tumor in patients with acromegaly. In a single-center series of 25 patients, Heck et al. reported that patients with hypointense had a significantly better biochemical response than patients with hyperintense adenomas after 6 months of first-line SSA therapy. In particular, IGF-I levels decreased by $51 \%$ in patients with hypointense and $13 \%$ in patients with hyperintense adenoma (16). Moreover, Potorac et al. evaluated a cohort of 120 acromegalic patients, finding that patients with hypointense adenomas had GH and IGF-I levels decrease of $88 \%$ and $59 \%$, respectively, whereas patients with hyperintense adenomas had GH and IGF-I levels decrease of $36.1 \%$ and $33.2 \%$, respectively, after 6 months of first-line SSA treatment with a significantly difference of these results between the two groups. Despite this evidence, normalization of IGF-I occurred in $25 \%$ of the hypointense group and in $37 \%$ of the hyperintense group, and normalization of both GH and IGF-I was reported in $16.7 \%$ of hypointense and $15.8 \%$ of hyperintense group, demonstrating absence of correlation between the T2-weighted signal intensity of the pituitary tumor and normalization of biochemical parameters of acromegaly, probably due to different baseline levels between the two groups of patients (14). Furthermore, quantitative assessment of $\mathrm{T} 2$-weighted signal intensity of the pituitary tumors was also employed in other studies, confirming that lower T2-weighted tumor signal intensity was associated with a better response in terms of GH and IGF-I reductions after 3 months $(36)$ and 6 months $(15,17)$ of SSA therapy. These two recent studies calculated predictive cut-offs of T2-weighted signal ratios for the biochemical efficacy of SSA treatment $(15,17)$. Potorac et al. did not find significant difference between the quantitative and qualitative evaluation of the $\mathrm{T} 2$-weighted tumor signal intensity as predictors of the biochemical response to SSA therapy (15). Heck et al. confirmed the accuracy of the qualitative assessment as well as the quantitative evaluation (17). 
However, the cut-off values proposed by the two different studies were not directly comparable, because of the employment of two different reference tissues for the assessment of the $\mathrm{T} 2$-weighted signal tumor intensity. Therefore, a definitive quantitative cut-off value of T2weighted tumor signal ratio, which is predictive of the biochemical response to SSA therapy, is far to be defined and used in clinical practice. The current study confirm a significantly greater reduction in tumor volume in patients with hypointense compared to patients with hyperintense adenomas on the T2-weighted images after 12 months of SSA therapy, confirming the greater tumor shrinkage associated with hypointense compared to hyperintense adenomas observed in previous studies $(14,17)$. Potorac et al. found significantly greater tumor shrinkage in hypointense $(37.8 \%)$ hyperintense (3\%) adenomas after 6 months of treatment (14). This evidence is in keeping with the findings of Heck et al. who showed that adenomas with lower T2weighted signal intensity had greater tumor shrinkage after median 6 months (17). Conversely, Shen et al. did not find any significant correlation between tumor volume reduction and T2-weighted tumor signal intensity, evaluating the predictive value of the signal intensity for the shortterm efficacy (3 months) of SSA therapy with a specific retrospective review of prospectively recorded data (37). The controversial results between the previous studies probably depend on the variable definition of the different classes of adenomas throughout the studies. Hagiwara $e t$ al. defined T2-hypointensity as a signal intensity lower than the white matter, whereas T2-hyperintensity as a signal intensity higher than the grey matter, and, consequently, T2-isointensity resulted to be the entire range of signal intensities between that of white and grey matter (5). This definition later employed by different studies $(13,16)$ places many adenomas in the isointense category. Different studies compared the tumor signal intensity only to that of white matter $(37,38)$. The definition of $\mathrm{T} 2$-weighted signal intensity used in different studies $(15,38)$ allows an easy visual classification of the adenomas, comparing adenoma intensity to that of normal pituitary tissue. In these studies, the grey matter of the temporal lobe was used as a comparator, when normal pituitary tissue is not visible. This is considered a logical choice, since the temporal lobe grey matter has a very similar signal intensity to that of normal pituitary tissue (15), therefore representing a useful standard reference. The current study adopted this latter methodology to evaluate the T2-intensity signal, since it is the simplest methodology and the most faithful to the corresponding pathological feature of the pituitary adenomas.

\section{Study limitations}

The study design is a retrospective cohort study, where different assays for the evaluation of GH and IGF-I were used, as part of the clinical routine evaluation, and dosage of SSA treatment was not predefined. The current study considered as the endpoint for medical treatment outcome a relative reduction of GH and IGF-I levels, whereas the biochemical control in the management of acromegaly is usually defined as the normalization of IGF-I levels adjusted for age and GH levels below $1 \mu \mathrm{g} / \mathrm{L}$. Moreover, the total number of patients included in the current study was relatively limited, and a further validation of these data should be confirmed in a larger, prospective study with a strict treatment protocol.

\section{Conclusions}

Pituitary gland MRI study can give the clinicians predictive information on the response to pharmacological therapy of GH secreting adenomas. With the use of T2 weighted sequences $\mathrm{GH}$-secreting adenomas can be classified into a hypointense group, which is associated to a better biochemical and radiological response to conventional SSA therapy, and hyperintense group, which is associated to a worse biochemical and radiological response to conventional SSA therapy.

\section{Acknowledgments}

None.

\section{Footnote}

Conflicts of Interest: The authors have no conflicts of interest to declare.

Ethical Statement: The authors are accountable for all aspects of the work in ensuring that questions related to the accuracy or integrity of any part of the work are appropriately investigated and resolved. This study does not require the approval from the ethics committee since the study is based on laboratory instrumental tests (biochemical evaluation of GH and IGF-I serum levels) and on radiological instrumental examinations (MRI exams of the pituitary gland) commonly used in the follow-up of 
patients with pituitary adenomas treated with somatostatin analogues.

\section{References}

1. Melmed S. Acromegaly pathogenesis and treatment. J Clin Invest 2009;119:3189-202.

2. Daly AF, Rixhon M, Adam C, et al. High prevalence of pituitary adenomas: a cross-sectional study in the province of Liege, Belgium. J Clin Endocrinol Metab 2006;91:4769-75.

3. Fernandez A, Karavitaki N, Wass JA. Prevalence of pituitary adenomas: a community-based, cross-sectional study in Banbury (Oxfordshire, UK). Clin Endocrinol (Oxf) 2010;72 377-82.

4. Petrossians P, Tichomirowa MA, Stevenaert A, et al. The Liege Acromegaly Survey (LAS): a new software tool for the study of acromegaly. Ann Endocrinol (Paris) 2012;73:190-201.

5. Hagiwara A, Inoue $\mathrm{Y}$, Wakasa K, et al. Comparison of growth hormone-producing and non-growth hormoneproducing pituitary adenomas: imaging characteristics and pathologic correlation. Radiology 2003;228:533-8.

6. Bonneville JF, Bonneville F, Cattin F. Magnetic resonance imaging of pituitary adenomas. Eur Radiol 2005;15:543-8.

7. Katznelson L, Laws ER Jr, Melmed S, et al. Endocrine Society. Acromegaly:an endocrine society clinical practice guideline. J Clin Endocrinol Metab 2014;99:3933-51.

8. Colao A, Grasso LFS, Giustina A, et al. Acromegaly. Nat Rev Dis Primers 2019 21;5:20.

9. Gadelha MR, Wildemberg LE, Bronstein MD, et al. Somatostatin receptor ligands in the treatment of acromegaly. Pituitary 2017;20:100-8.

10. Brzana J, Yedinak CG, Gultekin SH, et al. Growth hormone granulation pattern and somatostatin receptor subtype 2A correlate with postoperative somatostatin receptor ligand response in acromegaly:a large single center experience. Pituitary 2013;16:490-8.

11. Raverot G, Burman P, McCormack A, et al. European Society of Endocrinology Clinical Practice Guidelines for the management of aggressive pituitary tumours and carcinomas. Eur J Endocrinol 2018;178:G1-24.

12. Colao A, Grasso LF, Pivonello R, et al. Therapy of aggressive pituitary tumors. Expert Opin Pharmacother 2011;12:1561-70.

13. Puig-Domingo M, Resmini E, Gomez-Anson B, et al. Magnetic resonance imaging as a predictor of response to somatostatin analogs in acromegaly after surgical failure. J Clin Endocrinol Metab 2010;95:4973-8.

14. Potorac I, Petrossians P, Daly AF. T2-weighted MRI signal predicts hormone and tumor responses to somatostatin analogs in acromegaly. Endocr Relat Cancer 2016;23:871-81.

15. Potorac I, Petrossians P, Daly AF, et al. Pituitary MRI characteristics in 297 acromegaly patients based on T2weighted sequences. Endocr Relat Cancer 2015;22:169-77.

16. Heck A, Ringstad G, Fougner SL, et al. Intensity of pituitary adenoma on $\mathrm{T} 2$-weighted magnetic resonance imaging predicts the response to octreotide treatment in newly diagnosed acromegaly. Clin Endocrinol (Oxf) 2012;77:72-8.

17. Heck A, Emblem KE, Casar-Borota O, et al. Quantitative analyses of T2-weighted MRI as a potential marker for response to somatostatin analogs in newly diagnosed acromegaly. Endocrine 2016;52:333-43.

18. Go JL, Rajamohan AG. Imaging of the Sella and Parasellar Region. Radiol Clin North Am 2017;55:83-101.

19. Edal AL, Skjodt K, Nepper-Rasmussen HJ. SIPAP-a new MR classification for pituitary adenomas. Suprasellar, infrasellar, parasellar, anterior and posterior. Acta Radiol 1997;38:30-6.

20. Knosp E, Steiner E, Kitz K, Cet al. Pituitary adenomas with invasion of the cavernous sinus space:a magnetic resonance imaging classification compared with surgical findings. Neurosurgery 1993;33:610-7.

21. Katznelson L, Laws ER Jr, Melmed S, et al. Acromegaly: an endocrine society clinical practice guideline. J Clin Endocrinol Metab 2014;99:3933-51.

22. Chen CJ, Ironside N, Pomeraniec IJ, et al. Microsurgical versus endoscopic transsphenoidal resection for acromegaly: a systematic review of outcomes and complications. Acta Neurochir (Wien) 2017;159:2193-207.

23. Grasso, LF, Pivonello R, Colao A. Somatostatin analogs as a first- line treatment in acromegaly: when is it appropriate? Curr Opin Endocrinol Diabetes Obes 2012;19:288-94.

24. Grasso LF, Auriemma RS, Pivonello R, et al. Somatostatin analogs, cabergoline and pegvisomant: comparing the efficacy of medical treatment for acromegaly. Expert Rev Endocrinol Metab 2017;12:73-85.

25. Fleseriu M, Hoffman AR, Katznelson L. AACE Neuroendocrine and Pituitary Scientific Committee. American Association of Clinical Endocrinologists and American College of Endocrinology Disease State clinical review: management of acromegaly patients: what is the 
role of pre- operative medical therapy? Endocr Pract 2015;21:668-73.

26. Carlsen SM, Lund-Johansen M, Schreiner T, et al. Preoperative Octreotide treatment of acromegaly study group: preoperative octreotide treatment in newly diagnosed acromegalic patients with macroadenomas increases cure short-term postoperative rates: a prospective, randomized trial. J Clin Endocrinol Metab 2008;93:2984-90.

27. Mao ZG, Zhu YH, Tang HL, et al. Preoperative lanreotide treatment in acromegalic patients with macroadenomas increases short-term postoperative cure rates: a prospective, randomized trial. Eur J Endocrinol 2010;162:661-6.

28. Shen M, Shou X, Wang Y, et al. Effect of presurgical long-acting octreotide treatment in acromegaly patients with invasive pituitary macroadenomas: a prospective randomized study. Endocr J 2010;57:1035-44.

29. Bacigaluppi S, Gatto F, Anania P, et al. Impact of pre-treatment with somatostatin analogs on surgical management of acromegalic patients referred to a single center. Endocrine 2016;51:524-33.

30. Colao A, Auriemma RS, Pivonello R, et al. Interpreting biochemical control response rates with first- generation somatostatin analogues in acromegaly. Pituitary 2016;19:235-47.

31. Carmichael JD, Bonert VS, Nuño M, et al. Impact on reported biochemical efficacy rates of somatostatin

Cite this article as: Tortora F, Negro A, Grasso LFS, Colao A, Pivonello R, Splendiani A, Brunese L, Caranci F. Pituitary magnetic resonance imaging predictive role in the therapeutic response of growth hormone-secreting pituitary adenomas. Gland Surg 2019;8(Suppl 3):S150-S158. doi: 10.21037/ gs.2019.06.04 receptor ligand treatments:a meta- analysis. J Clin

Endocrinol Metab 2014;99:1825-33.

32. Colao A, Pivonello R, Galderisi M, et al. Impact of treating acromegaly first with surgery or somatostatin analogs on cardiomyopathy. J Clin Endocrinol Metab 2008;93:2639-46.

33. Colao A, Auriemma RS, Pivonello R. The effects of somatostatin analogue therapy on pituitary tumor volume in patients with acromegaly. Pituitary 2016;19:210-21.

34. Caron PJ, Bevan JS, Petersenn S, et al. Tumor shrinkage with lanreotide Autogel $120 \mathrm{mg}$ as primary therapy in acromegaly: results of a prospective multicenter clinical trial. J Clin Endocrinol Metab 2014;99:1282-90.

35. Colao A, Auriemma RS, Lombardi G, et al. Resistance to somatostatin analogs in acromegaly. Endocr Rev 2011;32:247-71.

36. Potorac I, Beckers A, Bonneville JF. T2-weighted MRI signal intensity as a predictor of hormonal and tumoral responses to somatostatin receptor ligands in acromegaly: a perspective. Pituitary 2017;20:116-20.

37. Shen M, Zhang Q, Liu W, et al. (2016) Predictive value of T2 relative signal intensity for response to somatostatin analogs in newly diagnosed acromegaly. Neuroradiology 2016;58:1057-65.

38. Bakhtiar Y, Hanaya R, Tokimura H, et al. Geometric survey on magnetic resonance imaging of growth hormone producing pituitary adenoma. Pituitary 2014;17:142-9. 